\title{
OCT in Alzheimer's disease: thinning of the RNFL and superior hemiretina
}

\author{
João Paulo Cunha ${ }^{1,2,3} \cdot$ Rita Proença $^{1} \cdot$ Arnaldo Dias-Santos $^{1} \cdot$ Rita Almeida $^{4}$. \\ Helena Águas ${ }^{4}$ - Marta Alves ${ }^{5}$ - Ana Luísa Papoila ${ }^{2,5,6}$ • Carlota Louro ${ }^{2}$. \\ António Castanheira-Dinis ${ }^{7}$
}

Received: 7 February 2017 /Revised: 30 May 2017 / Accepted: 5 June 2017 / Published online: 22 June 2017

(C) Springer-Verlag GmbH Germany 2017

\begin{abstract}
Background Peripapillary retinal nerve fiber layer (pRNFL) and internal macular layer thinning have been demonstrated in Alzheimer's disease (AD) with optical coherence tomography (OCT) studies. The purpose of this study is to compare the pRNFL thickness and overall retinal thickness (RT) in AD patients with non-AD patients, using spectral domain optical coherence tomography (SD-OCT) and determine the sectors most characteristically affected in AD.

Methods A cross-sectional study was performed to determine the pRNFL and overall macular RT thicknesses in AD and non-AD patients, attending a tertiary hospital center. For pRNFL, the global and six peripapillary quadrants were cal-
\end{abstract}

Electronic supplementary material The online version of this article (doi:10.1007/s00417-017-3715-9) contains supplementary material, which is available to authorized users.

João Paulo Cunha cunha.oft@gmail.com

1 Department of Ophthalmology, Central Lisbon Hospital Center, Lisbon, Portugal

2 NOVA Medical School, Universidade NOVA de Lisboa, Lisbon, Portugal

3 Department of Neuro-Ophthalmology, Central Lisbon Hospital Center, 1169-050 Lisboa, Portugal

4 Department of Neurology, Central Lisbon Hospital Center, Lisbon, Portugal

5 Epidemiology and Statistics Unit, Research Centre, Central Lisbon Hospital Center, Lisbon, Portugal

6 CEAUL (Center of Statistics and Applications), Lisbon University, Lisbon, Portugal

7 Visual Sciences Study Center, Faculty of Medicine, Lisbon University, Lisbon, Portugal culated, and for overall RT values, the nine Early Treatment Diabetic Retinopathy Study (ETDRS) areas were used. A multiple regression analysis was applied to assess the effects of disease, age, gender, spherical equivalent, visual acuity, intraocular pressure, axial length and blood pressure on pRNFL and overall macular RT.

Results A total of 202 subjects, including 50 eyes of 50 patients with mild $\mathrm{AD}$ (mean age 73.10; $\mathrm{SD}=5.36$ years) and 152 eyes of 152 patients without $\mathrm{AD}$ (mean age 71.03; $\mathrm{SD}=$ 4.62 years). After Bonferroni correction, the pRNFL was significantly thinner for the $\mathrm{AD}$ group globally and in the temporal superior quadrant $(10.76 \mu \mathrm{m}$ and $20.09 \mu \mathrm{m}$ mean decrease, respectively). The RT thickness was also decreased in superior sectors S3 and S6 (mean thinning of $9.92 \mu \mathrm{m}$ and $11.65 \mu \mathrm{m}$, respectively). Spearman's correlation coefficient showed a direct association between pRNFL in the temporal superior quadrant and RT in superior $\mathrm{S} 6$ and $\mathrm{S} 3$ sectors $\left(\mathrm{r}_{\mathrm{S}}=\right.$ $0.41 ; p<0.001$ and $\mathrm{r}_{\mathrm{S}}=0.28 ; p<0.001$, respectively).

Conclusions Patients with $\mathrm{AD}$ showed a significant thickness reduction in global and temporal superior quadrants in $\mathrm{pRNFL}$ and in superior pericentral and peripheral sectors of RT. These findings may reflect a peripapillary and retinal changes characteristic of $\mathrm{AD}$, suggesting the importance of SD-OCT as a potential adjuvant in early diagnosis of $\mathrm{AD}$. Further studies are needed to understand which retinal layers and macular sectors are more useful as potential ocular biomarker over time in $\mathrm{AD}$.

Keywords Alzheimer's disease - Spectral domain optical coherence tomography $\cdot$ RNFL $\cdot$ Retina $\cdot$ Macula

\section{Introduction}

Alzheimer's disease (AD) is the most common form of dementia in elderly with great social impact [1]. Due to the 
increase in human life expectancy, the prevalence of $\mathrm{AD}$ is also expected to follow the same trend and, consequently, the need for early diagnosis emerges. The earliest AD pathological change in the central nervous system is the accumulation of amyloid $\beta$, derived from the abnormal processing of amyloid precursor protein [2]. This process can begin a decade before the onset of the clinical syndrome of dementia. Visual symptoms are also frequent among the earliest complaints in $\mathrm{AD}$ patients, contributing to further impairment in the quality of life $[3,4]$.

Hinton et al. first provided histopathological evidence of optic neuropathy and degeneration of retinal ganglion cells (RGCs) in patients with $\mathrm{AD}$, with reduced number of RGCs and reduced retinal nerve fiber layer (RNFL) thickness [5, 6]. Later post-mortem studies showed that degeneration of the ganglion cell layer (GCL) occurs preferentially in superior and inferior quadrants, as well as in the central retina, in particular, the temporal foveal region $[7,8]$.

In vivo studies of optic neuropathy in patients with $\mathrm{AD}$ using fundus photographs showed RNFL and optic nerve head abnormalities (increased cup-to-disc ratio and decreased neuroretinal rim)[9-13], as well reduced macular thickness and volume [14-23].

The present study aimed to identify the quadrants in which peripapillary RNFL (pRNFL) and overall retinal thickness (RT) changes were more pronounced in patients with $\mathrm{AD}$, using spectral domain optical coherence tomography (SDOCT). This study also took into account potential confounding variables such as age, gender, spherical equivalent, best corrected visual acuity (BCVA), axial length, intraocular pressure (IOP), arterial blood pressure, therapy with diuretics and antihypertensive medication.

\section{Materials and Methods}

\section{Subject groups}

This cross-sectional study was conducted at the Ophthalmology and Neurology Departments of the Central Hospital Lisbon Center (CHLC), between October 2014 and April 2016. Consecutive AD patients sent by the Neurology Department for ophthalmological screening were observed for inclusion/exclusion criteria. Patients with clinical criteria for $\mathrm{AD}$ and mini-mental state examination (MMSE) scores between 21 and 26 were selected for the AD group (ADG) and subjects without clinical criteria for dementia and MMSE scores greater than 26 were included in the control group (CG).

The inclusion criteria were $\mathrm{AD}$ patients between 65 and 78 years old with normotensive eyes, and ability to understand the study.
Exclusion criteria were refractive error $>5$ diopters (D) or/ and axial length $>25 \mathrm{~mm}$ in the studied eye; known diagnosis of diabetes; retinal diseases; glaucoma or ocular hypertension; uveitis; neurodegenerative diseases and significant media opacities that precluded fundus imaging. Other relevant known neurologic pathology, such as neurodegenerative diseases, other types of dementia, previous stroke or uncertain or indeterminate diagnosis was excluded.

The study protocol was approved by the local ethical committee, patient's informed consent was obtained, and all the procedures were performed in accordance with the revised form of the Declaration of Helsinki (2008).

Fifty patients with mild AD (ADG) and 152 patients without AD (CG) were recruited from the Neurology Department of CHLC.

\section{Study Procedures}

After a pre-screening visit where demographic, background history, full ophthalmological examination with visual acuity, anterior segment examination, tonometry, indirect ophthalmoscopy and ultrasonic biometry were recorded, patients were assigned to a specific study visit where the following methodology was taken: Goldmann applanation tonometry and SD-OCT. Only the measurements of a randomly selected eye of each subject were used.

\section{Visual Acuity}

BCVA for each eye was measured using Snellen charts and converted to the logarithm of the minimum angle of resolution ( $\log M A R)$.

\section{Intraocular pressure}

IOP was measured before pupillary dilation with Goldmann applanation tonometry and a mean of three measurements was considered.

\section{Spectral Domain Optical Coherence Tomography Imaging}

SD-OCT (Spectralis Heidelberg Engineering, Germany, software version 6.0) was used for both eyes of each patient and performed in the same visit for peripapillary and macular observations. For pRNFL measurements, three consecutive scans were obtained using a circle size of $3.4 \mathrm{~mm}$, at $2.6^{\circ}$ nasal and $2.1^{\circ}$ superior off center. The software allows the mapping of thicknesses for the seven peripapillary quadrants (G - global, TS - temporal superior, T - temporal, TI - temporal inferior, NI - nasal inferior, N - nasal, and NS - nasal superior) according to Fig. 1. 

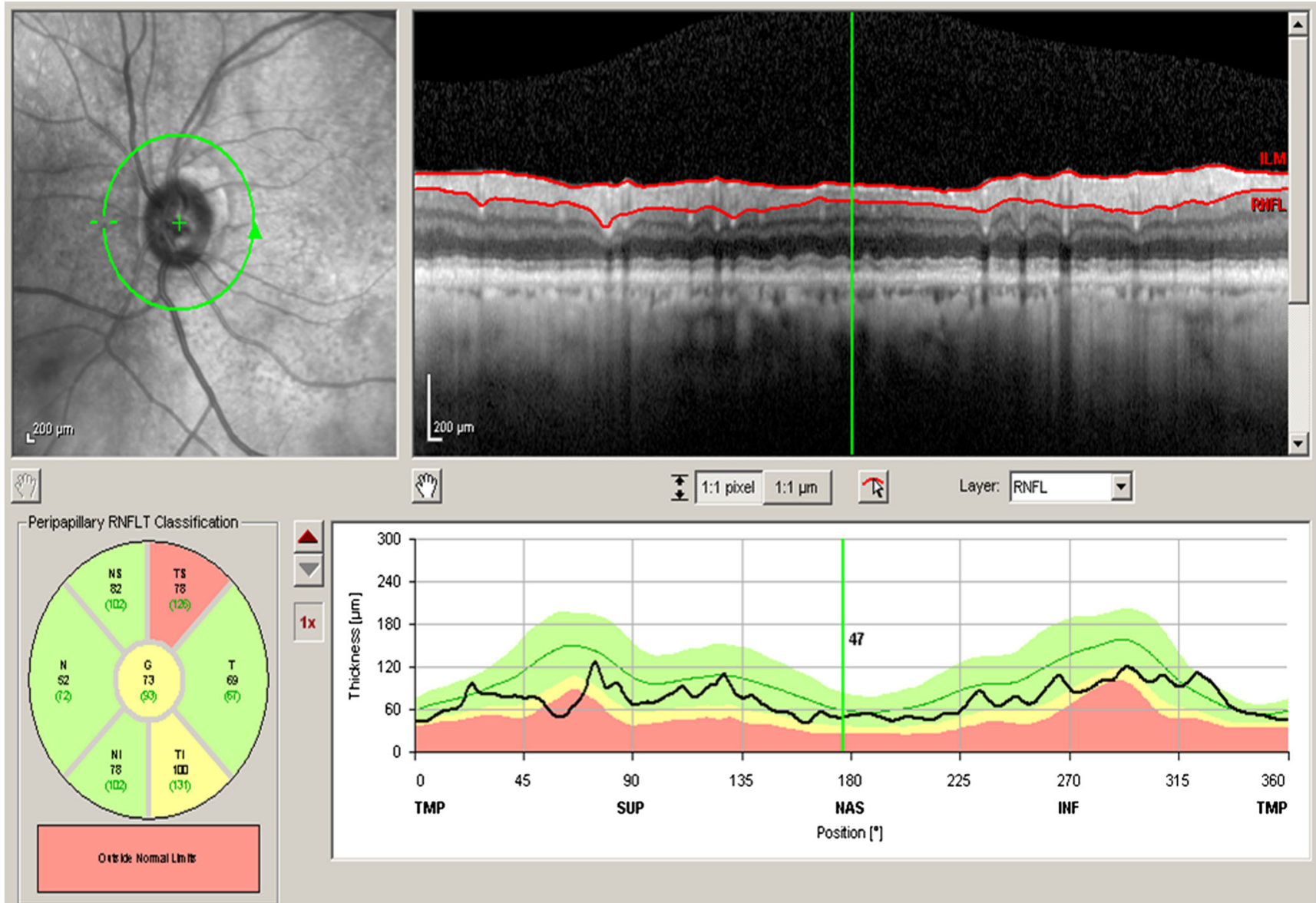

\section{[ing}

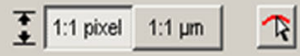

Layer: RNFL

$\checkmark$

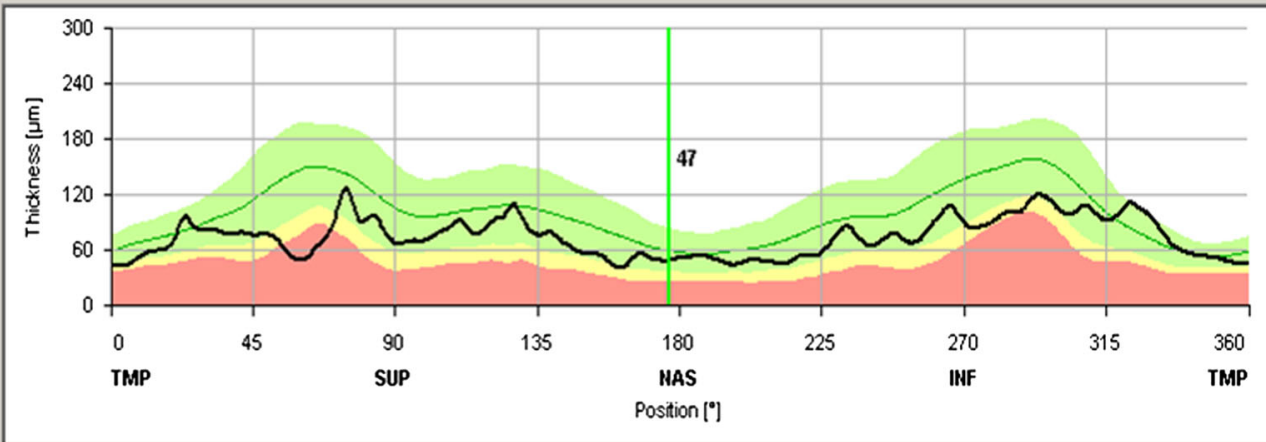

Fig. 1 Thickness of retinal nerve fiber layer obtained by "RNFL Single Exam Report OU with FoDi"M" (Spectralis Heidelberg; $\mu \mathrm{m})$.

For macular measurements, subjects were studied using the "fast macular volume" preset, consisting of a 25 -line horizontal raster scan covering $20^{\circ} \times 20^{\circ}$, centered on the fovea (consisting of 25 high-resolution scans). The overall RT values were calculated for the nine Early Treatment Diabetic Retinopathy Study (ETDRS) areas [24]. These ETDRS plots consist in three concentric rings of 1-, 3- and 6-mm diameter centered at the fovea with the two outer rings subdivided into four quadrants. Each sector was designated according to Fig. 2, the fovea or central sector (C), the pericentral ring (ETDRS sectors: S3, T3, I3 and N3) and the peripheral ring (ETDRS sectors: S6, T6, I6 and N6).

The OCT images were obtained by one ophthalmologist and were assessed by two other ophthalmologists, masked to the patients' diagnosis, who verified the automatic position of the ETDRS grid, correcting when necessary.

\section{Systolic and diastolic blood pressure}

Blood pressure was measured in the seated position by an automatic sphygmomanometer and systolic and diastolic blood pressure (SBP and DBP) were recorded. Mean arterial pressure (MAP) was calculated using the following formula:

$\mathrm{MAP}=\mathrm{DBP}+1 / 3(\mathrm{SBP}-\mathrm{DBP})$

\section{Statistical analysis}

An exploratory analysis was carried out for all variables. Categorical data were presented as frequencies (percentages), and continuous variables as mean and standard deviation (SD) or median and inter-quantile range (IQR: $25^{\text {th }}$ percentile $-75^{\text {th }}$ percentile), as appropriate. Nonparametric chi-square tests and Mann-Whitney tests were applied.

Linear regression models were used to identify the variables which may explain the variability of macular retinal and pRNFL thicknesses. The variables group, gender, age, IOP, axial length, spherical equivalent, MAP, BCVA, therapy with diuretics and antihypertensive medication, were considered in this analysis. In the univariable regression analysis, all the variables with a $p$ value $<0.25$ were selected for the multivariable models. Normality assumption of the residuals was verified using Kolmogorov-Smirnov goodness-of-fit test with Lilliefors correction. 

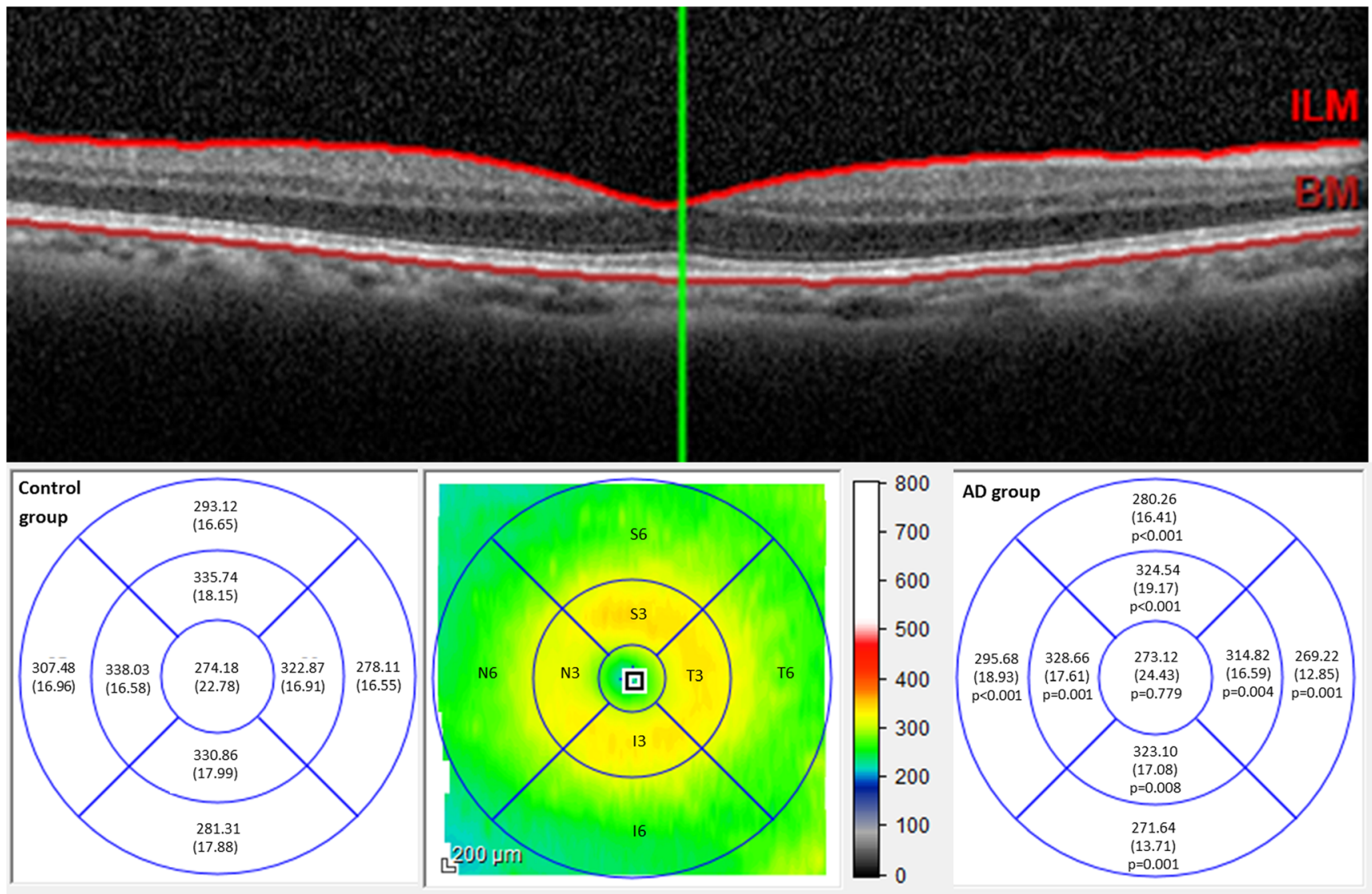

Fig. 2 Comparison of macular thickness $(\mu \mathrm{m})$ between control group and Alzheimer's disease (AD) group at nine sectors. Results are expressed as mean and standard deviation; $p$ values were obtained by univariable linear regression models.

To study the association between pRNFL quadrant thicknesses and overall RT of each sector, Spearman's correlation coefficient $\left(\mathrm{r}_{\mathrm{S}}\right)$ was used.

A level of significance of $\alpha=0.05$ was considered. Data were analyzed using the Statistical Package for the Social Sciences for Windows (IBM Corp. released 2013. IBM SPSS Statistics for Windows, Version 22.0. Armonk, NY: IBM Corp.).

\section{Results}

\section{Patient demographics and clinical characteristics}

A total of $50 \mathrm{AD}$ patients were included in the $\mathrm{AD}$ group and 152 patients without $\mathrm{AD}$ were included in the $\mathrm{CG}$. Concerning gender, no significant differences were found between $\mathrm{AD}$ and CGs (male $32.0 \%$ vs. $36.2 \%$; $p=0.591$ ). The mean age was $73.1(\mathrm{SD}=5.36)$ years in the $\mathrm{AD}$ group and $71.0(\mathrm{SD}=$ 4.62) years in the $\mathrm{CG}(p=0.011)$.

The demographic, clinical and ophthalmologic characteristics of the two groups, including BCVA, IOP, spherical equivalent, axial length, MAP, therapy with diuretics and antihypertensive medication, are summarized and compared in Table 1.

\section{OCT measurements of peripapillary retinal nerve fiber layer}

In a univariable analysis, the difference in thickness reached statistical significance in all sectors with a thinning of the pRNFL in the AD group compared with the CG (Table 2). The remaining univariable regression models for pRNFL thickness are presented in Supplemental Tables 1, 2 and 3.

In the multivariable linear regression models, after adjusting for factors such as age, gender, visual acuity, IOP, spherical equivalent, axial length, MAP, diuretic and antihypertensive medication, we have observed a global thinning of the pRNFL for the AD group in five of the seven peripapillary quadrants, including the nasal quadrants (NI $p=0.004 ; \mathrm{N} p=$ 0.004 and NS $p=0.004)$ and also $\mathrm{G}(p<0.001)$ and TS $(p<$ 0.001 ) quadrants (Table 3 ). The mean decrease of pRNFL in the $\mathrm{AD}$ group when compared with the $\mathrm{CG}$ was between $6.927 \mu \mathrm{m}$ and $20.089 \mu \mathrm{m}$. For each 10 years of increase of life, the mean values of the TS quadrant also decreased 
Table 1 Demographic and clinical characteristics of the patients by group

\begin{tabular}{llll}
\hline & $\begin{array}{l}\text { Alzheimer's group } \\
(\mathrm{n}=50)\end{array}$ & Control group (n=152) & $p$ value \\
\hline Age (years) & $73.1(5.36)$ & $71.0(4.62)$ & 0.011 \\
Male gender n (\%) & $16(32.0)$ & $55(36.2)$ & $0.591^{*}$ \\
BCVA (logMAR) & $0.121(0.153)$ & $0.040(0.073)$ & $<0.001$ \\
IOP - Goldmann (mmHg) & $15.52(2.62)$ & $14.72(2.51)$ & 0.066 \\
Spherical equivalent (D) & $0.995(1.43)$ & $0.700(1.64)$ & 0.344 \\
Axial length (mm) & $22.44(0.91)$ & $22.49(0.99)$ & 0.668 \\
Mean arterial pressure (mmHg) & $98.91(94.67-103.33)$ & $97.87(93.75-101.25)$ & 0.287 \\
Therapy with diuretics n (\%) & $14(29.8)$ & $26(17.1)$ & $0.058^{*}$ \\
Antihypertensive medication n (\%) & $32(64.0)$ & $60(39.5)$ & $0.003^{*}$ \\
\hline
\end{tabular}

Results are expressed as mean (SD) or median (IQR), as appropriate; best corrected visual acuity (BCVA); intraocular pressure (IOP). *Chi-square test; remaining $p$ values were obtained by Mann-Whitney tests.
$6.604 \mu \mathrm{m}(95 \%$ CI: -12.475 to -0.734$)$ (Table 3). Systolic blood pressure also remained in the multivariable models for nasal quadrants (NI $p=0.002 ; \mathrm{N} p=0.005$ and NS $p=0.048$ ) and also $\mathrm{G}(p=0.007)$, causing a mean thickening of the quadrants between $2.00 \mu \mathrm{m}$ and $4.89 \mu \mathrm{m}$, for each $10 \mathrm{mmHg}$ of increase. After Bonferroni correction, the quadrants of pRNFL still statistically thinner for AD group were localized in the $\mathrm{G}$ and TS quadrants $(10.755-\mu \mathrm{m}$ and 20.089- $\mu \mathrm{m}$ mean decrease, respectively).

\section{Retinal macular thickness}

The results of the univariable analysis showed that the RT was thinner in the AD group in eight of the nine sectors, between 1 and $6 \mathrm{~mm}$ centered at the fovea (Figure 2). The remaining univariable regression models for RT are presented in Supplemental Tables 4, 5 and 6.

In the multivariable linear regression models for seven RT sectors (no multiple model was achieved for the N6 sector), we observed a mean thinning in the AD group between

Table 2 Comparison of retinal nerve fiber layer (RFNL) at seven quadrants by group

\begin{tabular}{llll}
\hline Quadrants & $\begin{array}{l}\text { Alzheimer's group } \\
(\mathrm{n}=50)\end{array}$ & $\begin{array}{l}\text { Control group } \\
(\mathrm{n}=152)\end{array}$ & $p$ value \\
\hline Global & $85.72(14.42)$ & $96.51(9.36)$ & $<0.001$ \\
Temporal superior & $108.20(28.35)$ & $129.59(17.16)$ & $<0.001$ \\
Temporal & $64.22(13.01)$ & $71.38(12.66)$ & 0.001 \\
Temporal inferior & $120.68(23.69)$ & $136.69(20.06)$ & $<0.001$ \\
Nasal inferior & $99.40(25.95)$ & $110.00(22.22)$ & 0.006 \\
Nasal & $68.76(16.99)$ & $74.77(13.73)$ & 0.012 \\
Nasal superior & $93.14(29.08)$ & $103.50(17.45)$ & 0.003 \\
\hline
\end{tabular}

Results are expressed as mean (SD); $p$ values were obtained by univariable linear regression models.
$6.820 \mu \mathrm{m}$ and $11.649 \mu \mathrm{m}(p<0.001$ to 0.012$)$. A mean thinning between $4.714 \mu \mathrm{m}$ and $7.237 \mu \mathrm{m}$ for each 10 years of age increase ( $p=0.003$ to 0.046 ) was also observed in the multivariable models for sectors T3, N3, S6, T6 and I6.

The results of the multivariable regression models after Bonferroni correction showed two sectors still thinner with statistical significance: the pericentral and peripheral superior sectors S3 and S6 (mean thinning of $9.916 \mu \mathrm{m}$ and 11.649 $\mu \mathrm{m}$, with $p=0.001$ and $p<0.001$, respectively; Table 4). Also, a mean thickening of $15.676 \mu \mathrm{m}$ at the $\mathrm{C}$ sector in the RT for male gender was observed (Table 4).

Finally, Spearman's correlation coefficient showed a direct association between pRNFL TS quadrant and RT S6 sector $\left(\mathrm{r}_{\mathrm{S}}\right.$ $=0.41 ; p<0.001)$, stronger than the correlation between pRNFL TS quadrant and RT S3 sector $\left(\mathrm{r}_{\mathrm{S}}=0.28 ; p<0.001\right)$.

\section{Discussion}

Regarding RNFL thinning in AD, it has been hypothesized that the neuroretinal atrophy may occur as a result of amyloid $\beta$ plaque deposits within the retina [15] or as a result of retrograde degeneration of the RGC axons [17], and these changes have been suggested to occur even before memory is affected [18].

Since Parisi et al., pRNFL thinning has been demonstrated with time domain OCT (TD-OCT); however, differences have been reported regarding which peripapillary quadrants are most affected using TD- and/or SD-OCT [15, 17, 18, 25].

Whether or not a correlation exists between retinal changes and severity of dementia also remains a controversial issue. While most studies concluded that OCT could be used to detect early abnormalities in mild cognition impairment (MCI) and AD, the majority reported no statistically significant differences between MCI and AD patient groups [17, 26-29]. Only one TD-OCT study reported correlation 
Table 3 Results of multivariable regression models - dependent variable: RNFL thickness

\begin{tabular}{llll}
\hline Model & Coefficient estimate & $p$ value & $95 \%$ Confidence interval \\
\hline Dependent variable: RNFL thickness G & & & -14.162 \\
$\quad$ Alzheimer's group* & -10.755 & $<0.001 \S$ & -6.485 \\
Male gender & -3.433 & 0.028 & 0.056 \\
Systolic blood pressure & 0.200 & 0.007 & -7.349 \\
Dependent variable: RNFL thickness TS & & & -26.710 \\
Alzheimer's group* & -20.089 & $<0.001 \S$ & -12.475 \\
Age (years) & -6.604 & 0.028 & -18.394 \\
Dependent variable: RNFL thickness NI & & & -14.007 \\
Alzheimer's group* & -11.017 & 0.004 & 0.177 \\
Male gender & -7.393 & 0.029 & -13.468 \\
Systolic blood pressure & 0.489 & 0.002 & -0.734 \\
Dependent variable: RNFL thickness N & & & -3.635 \\
Alzheimer's group* & -6.927 & 0.004 & -0.778 \\
Age (years) & 4.415 & 0.037 & 0.270 \\
Systolic blood pressure & 0.283 & 0.005 & 0.086 \\
Dependent variable: RNFL thickness NS & & 0.004 & -2.212 \\
Alzheimer's group* & -9.990 & 0.048 & -16.774 \\
Systolic blood pressure & 0.290 & 0.003 & 0.480 \\
\hline
\end{tabular}

${ }^{*}$ Reference category: control group; age: for each increase of 10 years; $p$ values were obtained by linear regression models. $\S$ with statistical significance after Bonferroni correction.

between MMSE scores and macular volume [14]. Also, metaanalyses tried to determine the utility of OCT as a tool for evaluating disease progression, and prognostic significance of macular and RNFL thickness, but their conclusions failed to determine a correlation between RNFL and the clinical severity of dementia [30-34].

In addition, one study using SD-OCT showed a diffuse reduction of the RNFL and ganglion cell layer combined in AD [21] although the authors were not able to determinate which layer was most affected by AD. Others studies have demonstrated inner plexiform layer thinning in $\mathrm{AD}$ patients $[14,15$, $21,22,35]$. In a recent study analysing SD-OCT retinal markers, including RNFL thickness, GCL thickness did not show differences between $\mathrm{AD}$ patients age- and sex-matched controls or other neurodegenerative diseases, but the authors hypothesize that a larger sample would be necessary to delineate significant differences between the groups studied [36].

In this study, we used SD-OCT to compare pRNFL thickness and overall RT in mild AD patients with a large CG. In the multivariable analysis, after adjustment for age, gender, BCVA, IOP, axial length, spherical equivalent and MAP, the global and temporal quadrants of pRNFL, and superior pericentral and peripheral sectors of the overall retina were thinner. Spearman's coefficient showed a stronger correlation between TS quadrant and S6 sector than between TS quadrant and S3 sector. Our study shows that the classically described macular asymmetry is confirmed only when we compare nasal sectors with temporal ones.
After Bonferroni correction, the thinning was most pronounced in the superiors sectors of RT (S3 and S6), with the classically described superior/inferior asymmetry disappearing. Our results are consistent with the visual field findings reported by Trick et al. [37] and histopathological findings of Armstrong [38]. They predominantly observed inferior visual field defects and greater density of senile plaques and neurofibrillary tangles in the cuneal gyrus than in the lingual gyrus in patients with AD. The precuneus was also described as particularly vulnerable for AD pathology, including cerebral atrophy and amyloid deposition [39]. The axons from the superior retina project via the parietal lobe portion of the optic radiation to the cuneal gyrus of the primary visual cortex, and some authors found decreased longitudinal functional connectivity between the precuneus and other cerebral area networks in AD [40-42].

Our study had some limitations. The first one was the age distribution of the two study groups that was corrected by the linear regression models. Second, the automatic centration of the ETDRS grid could have resulted in imprecise measurements, but was confirmed by two independent ophthalmologists. Thirdly, although all participants were recruited from the Neurology Department with clinical criteria and MMSE, no amyloid markers (cerebrospinal fluid or amyloid imaging) were used to increase the accuracy of the diagnosis. Fourthly, as not all patients performed visual fields, the exclusion of glaucomatous disease was based on IOP values and indirect ophthalmoscopy. Lastly, like all cross-sectional 
Table 4 Results of multivariable regression models - dependent variable: RT thickness

\begin{tabular}{|c|c|c|c|c|}
\hline Model & $\begin{array}{l}\text { Coefficient } \\
\text { estimate }\end{array}$ & $p$ value & $95 \%$ Confidence interval & \\
\hline \multicolumn{5}{|c|}{ Dependent variable: $\mathrm{RT}$ thickness $\mathrm{C}$} \\
\hline Male gender & 15.676 & $<0.001 \S$ & 9.359 & 21.992 \\
\hline Axial lenghtl & 3.782 & 0.018 & 0.663 & 6.901 \\
\hline \multicolumn{5}{|c|}{ Dependent variable: RT thickness S3 } \\
\hline Alzheimer's group* & -9.916 & $0.001 \S$ & -15.852 & -3.980 \\
\hline Age (years) & -6.522 & 0.015 & -11.784 & -1.259 \\
\hline \multicolumn{5}{|c|}{ Dependent variable: RT thickness T3 } \\
\hline Alzheimer's group* & -6.820 & 0.012 & -12.133 & -1.508 \\
\hline Age (years) & -7.237 & 0.003 & -11.954 & -2.520 \\
\hline Male gender & 5.531 & 0.022 & 0.808 & 10.254 \\
\hline Spherical & 1.478 & 0.042 & 0.055 & 2.902 \\
\hline \multicolumn{5}{|c|}{ Dependent variable: RT thickness I3 } \\
\hline Alzheimer's group* & -8.231 & 0.005 & -13.916 & -2.546 \\
\hline Spherical & 1.630 & 0.038 & 0.091 & 3.170 \\
\hline \multicolumn{5}{|c|}{ Dependent variable: RT thickness N3 } \\
\hline Alzheimer's group* & -8.734 & 0.002 & -14.161 & -3.307 \\
\hline Age (years) & -5.389 & 0.029 & -10.211 & -0.567 \\
\hline Spherical & 1.456 & 0.050 & 0.001 & 2.910 \\
\hline \multicolumn{5}{|c|}{ Dependent variable: RT thickness S6 } \\
\hline Alzheimer's group* & -11.649 & $<0.001 \S$ & -16.995 & -6.304 \\
\hline Age (years) & -6.126 & 0.012 & -10.865 & -1.387 \\
\hline \multicolumn{5}{|c|}{ Dependent variable: RT thickness T6 } \\
\hline Alzheimer's group* & -7.955 & 0.002 & -13.048 & -2.861 \\
\hline Age (years) & -4.714 & 0.041 & -9.230 & -0.198 \\
\hline \multicolumn{5}{|c|}{ Dependent variable: RT thickness I6 } \\
\hline Alzheimer's group* & -8.692 & 0.002 & -14.186 & -3.197 \\
\hline Age (years) & -4.953 & 0.046 & -9.824 & -0.082 \\
\hline
\end{tabular}

* Reference category: control group; age: for each increase of 10 years; $p$ values were obtained by linear regression models. $\S$ with statistical significance after Bonferroni correction.

studies, it was not possible to draw conclusions about changes in pRNFL and RT in a single individual over time. As only the overall RT was considered, the atrophy of some retinal layers may only be compensated by others in an attempt to fill/ compensate the possible functional and/or structural consequences. In this sense, several studies showed that macular GC-IPL thinning may be a more sensitive marker of earlier neurodegeneration in MCI and AD than evaluation of the overall RT [26-29]. The next step is to understand which sectors of which layers can be earlier affected by AD and the possible confounding factors associated with the thinning of the layers.

\section{Conclusion}

Patients with $\mathrm{AD}$ showed a significant thickness reduction in global and temporal quadrants in the pRNFL and in the superior sector of the macula. This thinning may reflect a peripapillary and retinal characteristic of $\mathrm{AD}$, suggesting the importance of SD-OCT as a potential adjuvant in early diagnosis of AD. Further studies are needed to understand which retinal layers and macular sectors are more useful as potential clinical ocular biomarkers for early detection of $\mathrm{AD}$, and over time in disease progression.

Acknowledgements We would like to thank Bruno Oliveira-Santos for the helpful cooperation in collecting the OCT data. We thank Dr. Paula Mota and Dr. Joana Tavares-Ferreira for the detailed reading and comments on the manuscript.

\section{Compliance with ethical standards}

Funding No funding was received for this research.

Conflict of Interest All authors certify that they have no affiliationswith or involvement in any organization or entity with any financial interest (such as honoraria; educational grants; participation in 
speakers' bureaus; membership, employment, consultancies, stock ownership, or other equity interest; and expert testimony or patent-licensing arrangements), or non-financial interest (such as personal or professional relationships, affiliations, knowledge or beliefs) in the subject matter or materials discussed in this manuscript.

Ethical approval All procedures performed in studies involving humanparticipants were in accordance with the ethical standards of the institutional and/or national research committee and with the 1964 Helsinki Declaration and its later amendments or comparable ethical standards.

Informed consent Informed consent was obtained from all individualparticipants included in the study.

Financial disclosure None of the authors have any conflict of interest.

\section{References}

1. Association A (2012) 2012 Alzheimer's disease facts and figures. Alzheimer's Dementia 8:131-168. doi:10.1016/j.jalz.2012.02.001

2. Perl DP, Perl DP (2010) Neuropathology of Alzheimer 's Disease Address Correspondence to : $32-42$. doi: 10.1002/MSJ

3. Burns A, Iliffe S (2009) Alzheimer's disease. BMJ 338:b158. doi: 10.1136/bmj.b158

4. Querfurth HW, LaFerla FM (2010) Alzheimer's disease. N Engl J Med 362:329-344. doi:10.1056/NEJMra0909142

5. Hinton DR, Sadun AA, Blanks JC, Miller CA (1986) Optic-nerve degeneration in Alzheimer's disease. N Engl J Med 315:485-487. doi:10.1056/NEJM198608213150804

6. Hinton DR, Sadun AA, Blanks JC, Miller CA (2010) Optic nerve degeneration in Alzheimer's disease. N Engl J Med 315:485-487

7. Blanks JC, Torigoe Y, Hinton DR, Blanks RHI (1996) Retinal pathology in Alzheimer's disease. I. Ganglion cell loss in foveal/ parafoveal retina. Neurobiol Aging 17:377-384. doi:10.1016/ 0197-4580(96)00010-3

8. Blanks JC, Hinton DR, Sadun AA, Miller CA (1989) Retinal ganglion cell degeneration in Alzheimer's disease. Brain Res 501:364372

9. Parisi V, Restuccia R, Fattapposta F, et al (2001) Morphological and functional retinal impairment in Alzheimer's disease patients. 112: $1860-1867$

10. Berisha F, Feke GT, Trempe CL, et al (2007) Retinal Abnormalities in Early Alzheimer's Disease. 48:6-10. doi: 10.1167/iovs.06-1029

11. Hedges TR, Perez Galves R, Speigelman D et al (1996) Retinal nerve fiber layer abnormalities in Alzheimer's disease. Acta Ophthalmol Scand 74:271-275

12. Tsai C, Ritch R, Schwartz B et al (1991) Optic nerve head and nerve fiber layer in Alzheimer's disease. Arch Ophthalmol 109:199-204

13. Danesh-Meyer HV, Birch H, Ku JY et al (2006) Reduction of optic nerve fibers in patients with Alzheimer disease identified by laser imaging. Neurology 67:1852-1854

14. Iseri PK, Tokay T (2006) Relationship between Cognitive Impairment and Retinal Morphological and Visual Functional Abnormalities in Alzheimer Disease. 26:18-24

15. Kirbas S, Turkyilmaz K, Anlar O et al (2013) Retinal nerve fiber layer thickness in patients with Alzheimer disease. J Neuroophthalmol 33:58-61. doi:10.1097/WNO. 0b013e318267fd5f

16. Salobrar-garcia E, Hoz R De, Rojas B, et al (2015) Findings in Mild Alzheimer's Disease. 2015:17-19. doi: 10.1155/2015/736949

17. Ascaso FJ, Cruz N, Modrego PJ, Cristo A (2014) Retinal alterations in mild cognitive impairment and Alzheimer's disease : an optical coherence tomography study. 1522-1530. doi: $10.1007 / \mathrm{s} 00415-$ 014-7374-Z

18. Moschos M, Markopoulos I, Chatziralli I et al (2012) Structural and functional impairment of the retina and optic nerve in Alzheimer's disease. Curr Alzheimer Res 9:782-788. doi:10.2174/ 156720512802455340

19. Gao L, Liu Y, Li X et al (2015) Abnormal retinal nerve fiber layer thickness and macula lutea in patients with mild cognitive impairment and Alzheimer's disease. Arch Gerontol Geriatr 60:162-167. doi:10.1016/j.archger.2014.10.011

20. Williams MA, Mcgowan AJ, Cardwell CR et al (2015) Retinal microvascular network attenuation in Alzheimer' $\mathrm{s}$ disease. Alzheimer's Dement Diagnosis, Assess Dis Monit 1:229-235. doi:10.1016/j.dadm.2015.04.001

21. Marziani E, Pomati S, Ramolfo P, et al (2016) Evaluation of Retinal Nerve Fiber Layer and Ganglion Cell Layer Thickness in Alzheimer's Disease Using Spectral- Domain Optical Coherence Tomography. doi: 10.1167/iovs.13-12046

22. Ong Y, Ong Y, Ikram MK, et al (2014) Potential Applications of Spectral-Domain Optical Coherence Tomography ( SD-OCT ) in the Study of Alzheimer's Disease. 23:74-83

23. Garcia-Martin ES, Rojas B, Ramirez AI et al (2014) Macular Thickness as a Potential Biomarker of Mild Alzheimer's Disease. Ophthalmology 121:1149-1151.e3. doi:10.1016/j.ophtha.2013.12. 023

24. Group ETDRSR (1991) Early Photocoagulation for Diabetic Retinopathy. Ophthalmology 98:766-785. doi:10.1016/S01616420(13)38011-7

25. Salobrar-Garcia E, Hoyas I, Leal M, et al (2015) Analysis of Retinal Peripapillary Segmentation in Early Alzheimer's Disease Patients. doi: $10.1155 / 2015 / 636548$

26. Ozdemir E, Eda O, Seda D (2015) The relationship between the degree of cognitive impairment and retinal nerve fiber layer thickness. Neurol Sci:1141-1146. doi:10.1007/s10072-014-2055-3

27. Bambo MP, Garcia-Martin E, Pinilla J et al (2014) Detection of retinal nerve fiber layer degeneration in patients with Alzheimer's disease using optical coherence tomography: searching new biomarkers. Acta Ophthalmol 92:e581-e582. doi:10.1111/aos.12374

28. Paquet C, Roger F, Dighiero P, et al (2007) Abnormal retinal thickness in patients with mild cognitive impairment and Alzheimer's disease. 420:97-99. doi: 10.1016/j.neulet.2007.02.090

29. Kesler A, Vakhapova V, Korczyn AD et al (2011) Retinal thickness in patients with mild cognitive impairment and Alzheimer' s disease. Clin Neurol Neurosurg 113:523-526. doi:10.1016/j.clineuro. 2011.02.014

30. Tzekov R, Mullan M (2014) Vision function abnormalities in Alzheimer disease. Surv Ophthalmol 59:414-433. doi:10.1016/j. survophthal.2013.10.002

31. Coppola G, Renzo A Di, Ziccardi L, Martelli F (2015) Optical Coherence Tomography in Alzheimer's Disease : A MetaAnalysis. 1-14. doi: 10.1371/journal.pone.0134750

32. Cunha JP, Moura-Coelho N, Proença RP, et al (2016) Alzheimer's disease: A review of its visual system neuropathology. Optical coherence tomography - a potential role as a study tool in vivo. Graefe's Arch Clin Exp Ophthalmol. doi: 10.1007/s00417-0163430-y

33. Thomson KL, Yeo JM, Waddell B et al (2015) A systematic review and meta-analysis of retinal nerve fiber layer change in dementia, using optical coherence tomography. Alzheimer's Dement Diagnosis, Assess Dis Monit 1:136-143. doi:10.1016/j.dadm. 2015.03.001

34. He X-F, Liu Y-T, Peng C et al (2012) Optical coherence tomography assessed retinal nerve fiber layer thickness in patients with Alzheimer's disease: A meta-analysis. Int J Ophthalmol 5:401405. doi:10.3980/j.issn.2222-3959.2012.03.30 
35. Cheung CY, Ting Y, Ikram MK et al (2014) Microvascular network alterations in the retina of patients with Alzheimer ' s disease. Alzheimer's Dement 10:135-142. doi:10.1016/j. jalz.2013.06.009

36. Pillai JA, Bermel R, Bonner-Jackson A, et al (2016) Retinal Nerve Fiber Layer Thinning in Alzheimer's Disease: A Case-Control Study in Comparison to Normal Aging, Parkinson's Disease, and Non-Alzheimer's Dementia. Am J Alzheimers Dis Other Demen. doi: $10.1177 / 1533317515628053$

37. Trick GL, Trick LR, Morris P, Wolf M (1995) Visual field loss in senile dementia of the Alzheimer's type. Neurol 45:68-74. doi:10. 1212/WNL.45.1.68

38. Armstrong RA (1996) Visual field defects in Alzheimer's disease patients may reflect differential pathology in the primary visual cortex. Optom Vis Sci 73:677-682
39. Jack CR, Knopman DS, Jagust WJ et al (2010) Hypothetical model of dynamic biomarkers of the Alzheimer's pathological cascade. Lancet Neurol 9:119-128. doi:10.1016/S1474-4422(09)70299-6

40. Damoiseaux JS, Prater KE, Miller BL, Greicius MD (2012) Functional connectivity tracks clinical deterioration in Alzheimer' $\mathrm{s}$ disease. NBA 33:828.e19-828.e30. doi:10.1016/j.neurobiolaging. 2011.06.024

41. Hafkemeijer A, Möller C, Dopper EGP, Jiskoot LC (2015) Resting state functional connectivity differences between behavioral variant frontotemporal dementia and Alzheimer's disease. 9:1-12. doi: 10. 3389/fnhum.2015.00474

42. Hafkemeijer A, Christiane M (2017) A Longitudinal Study on Resting State Functional Connectivity in Behavioral Variant Frontotemporal Dementia and Alzheimer' s Disease. 55:521537. doi: 10.3233/JAD-150695 